\title{
Cohort-Specific Online Discussion Experiences: A Collaborative And Multidisciplinary Approach To Improving Student Learning
}

\author{
Adam L. Selhorst, Ashford University, USA \\ Eric Klein, Ashford University, USA \\ Justin Harrison, Ashford University, USA
}

\begin{abstract}
Research addressing the effects of cohort size on student success in asynchronous online discussions is sparse. As such, the following study attempted to determine an optimal student cohort size to enhance success and engagement within online discussions in general education courses at a large post-secondary university consisting of predominately adult learners. Experimental courses split mandatory discussions into one, two, or three cohorts to maintain a discussion size of no more than ten students per cohort per week. The effects of cohort size on student grade-point-average (GPA), withdraw rate, fail rate, and progression rate was evaluated in addition to effects on student satisfaction as measured by end-of-course surveys (EoCS). Results showed no significant difference in either student success or student satisfaction between courses with one, two, or three online discussion cohorts. Future online education research should focuses on upper division courses where students might benefit from smaller group discussions.
\end{abstract}

Keywords: Online Education; Adult Learners; Online Discussions; Cohort Size, Distance Education

\section{INTRODUCTION}

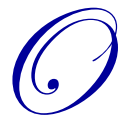

nline learning in higher education has been proliferating over the past two decades, and research related to best practices in teaching and learning continues to emerge at an exponential rate. Also, colleges and universities are being encouraged to develop, implement, and assess innovative, multidisciplinary approaches to improve student learning and success. One of the more prevalent and established methods of engaging students and enhancing learning in the online modality are asynchronous discussions, a tool that allows students to participate in the same discourse at different times.

Online discussion forums can create a substantial venue for dialogue, interaction, and the collaborative construction of knowledge. Furthermore, researchers and educators commonly accept that asynchronous discussions can enhance online learning and teaching. In online education, asynchronous discussions are commonly facilitated through learning management systems, which commonly includes tools such as a grade book and asynchronous discussion forums. While the advantages and limitations of utilizing asynchronous discussions have been well-documented, the specific success factors related to student achievement of learning outcomes are still fairly ambiguous.

For example, a comprehensive review of the literature suggests a lack of consensus related to the ideal size of online discussion groups. Much of the existing research suggests that the optimal online discussion group size ranges from approximately 4 - 9 students (Berry, 2008; Du, Durrington, \& Matthews, 2007; Schellens \& Valcke, 2006 Levin, He, \& Robbins, 2006), yet there is additional research that indicates both larger (Fisher, Thompson, \& Silverberg, 2005) and smaller (Fernandez, 2007; Huang \& Law, 2004) group size may be optimal. In general, online courses with larger class sizes are recognized as less individualized and more impersonal, as several studies have found larger class sizes 
negatively associated with student learning and satisfaction (e.g., Arbaugh \& Benbunan-Fich, 2006; Buckingham, 2003; Burruss, Billings, Brownrigg, Skiba, \& Connors, 2009; Schellens \& Valcke, 2006).

Moreover, the overall research on the effect of cohort-specific online discussion experiences is very limited. Specifically, despite the propagation of online higher education research, scant research exists regarding the impact of varying class or cohort sizes on student learning and engagement in online courses. Conclusions into appropriate class and discussion sizes (i.e., number of students per faculty member) are seemingly mixed and often based on anecdotal evidence. The primary research conclusion to this point is that altering class or cohort sizes does impact group dynamics and student-faculty relationships (e.g., Arbaugh \& Benbunan-Fich, 2006; Dykman, \& Davis, 2008; Schellens \& Valcke, 2006). As course and discussion sizes increase, students tend to experience less direct individual contact with faculty, and their satisfaction typically decreases. Moreover, faculty may perceive that the quality of education declines as they have less personalized interaction with students and diminished opportunities to engage with individual student needs.

Furthermore, the quality of student-faculty interaction has been found to be a strong and consistent predictor of student learning and student satisfaction, and faculty immediacy (i.e., personal and timely responsiveness) is one of the strongest predictors of student satisfaction (Keeton, 2004; Schutt, Allen, \& Laumakis, 2009). Also, the quality of student-student interaction has also been found to be predictive of learning (Keeton, 2004; Marks, Sibley, \& Arbaugh, 2005).

The purpose of this study was to explore whether student success and engagement could improve in asynchronous online discussion boards by optimizing the number of students in each discussion. Based on prior literature in conjunction with the expertise of the researchers, it was hypothesized that breaking larger class sizes into smaller discussion sections would have a positive effect on student success and satisfaction.

\section{METHODS}

\section{Student Demographics}

The study utilizes data gathered from undergraduate students taking ENG121 - English Composition I, ENG122 English Composition II, PHI103 - Informal Logic, POL210 - American National Government, SCI207 - Dependence of Man on the Environment, and SOC102 - Introduction to Ethics and Social Responsibility between January 5, 2016 and February 29, 2016 at Ashford University. Ashford University is a large post-secondary institution offering Associate's (2 Programs), Bachelor's (53 Programs), and Master's (11 Programs) degrees fully online. The student population consists largely of adult learners (Figure 1), with a disproportionate amount of female (70\%) to male (30\%) students, from a wide range backgrounds (Figure 2). Furthermore, military students make up $27 \%$ of the total enrollment as of the Fall of 2015 (Institutional Research Services, 2015). Additional information surrounding current student demographics can be obtained at http://assessment.ashford.edu/behind-numbers/institutional-data/enrolledstudent-characteristics\#/28/385. Due to the large sample size and a variety of courses, the student sample for this study should largely mirror the overall population of the university. 
Figure 1. Total enrollment by age range for Ashford University students as of Fall 2015

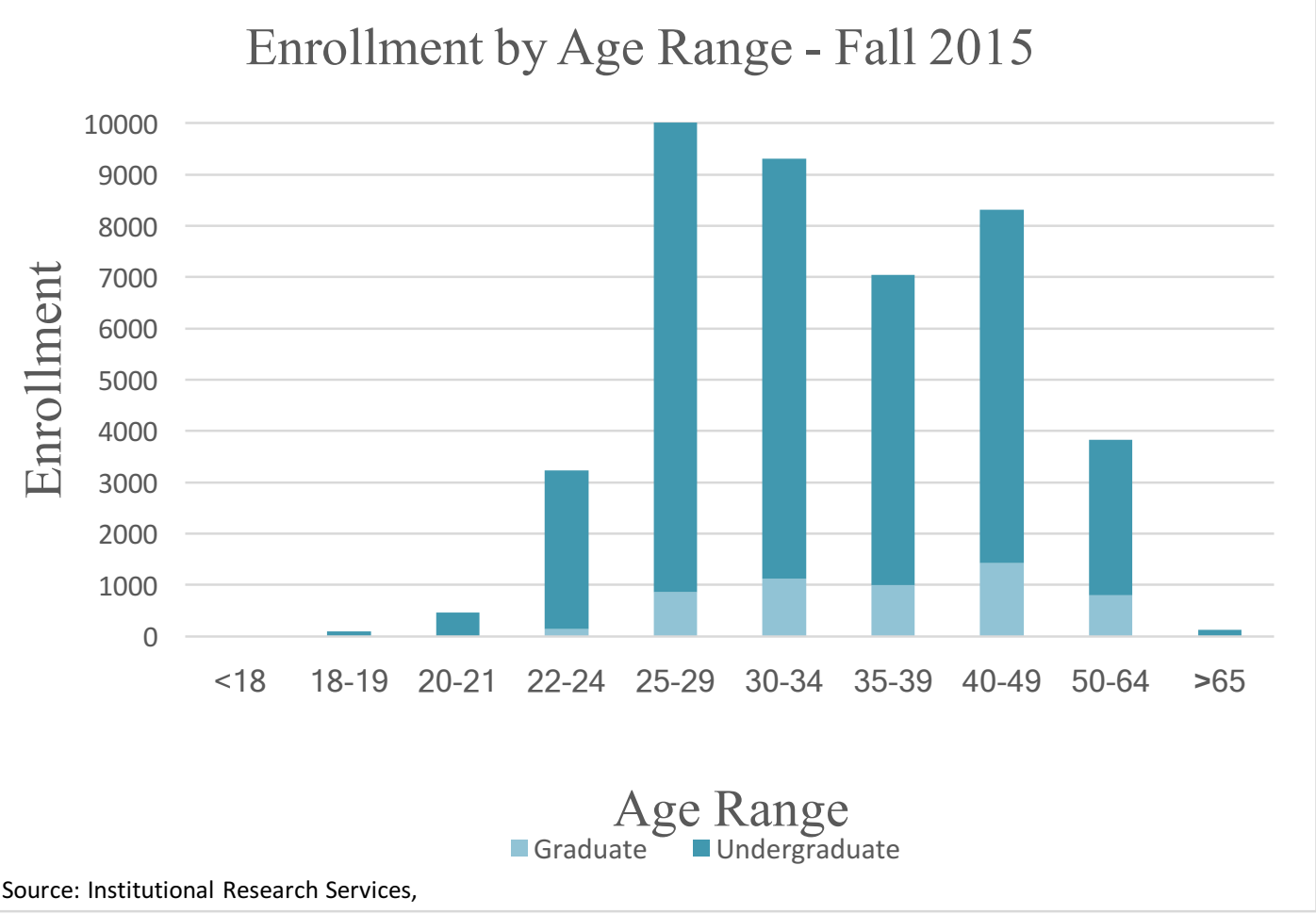

Figure 2. Total enrollment by race/ethnicity for Ashford University students as of Fall 2015

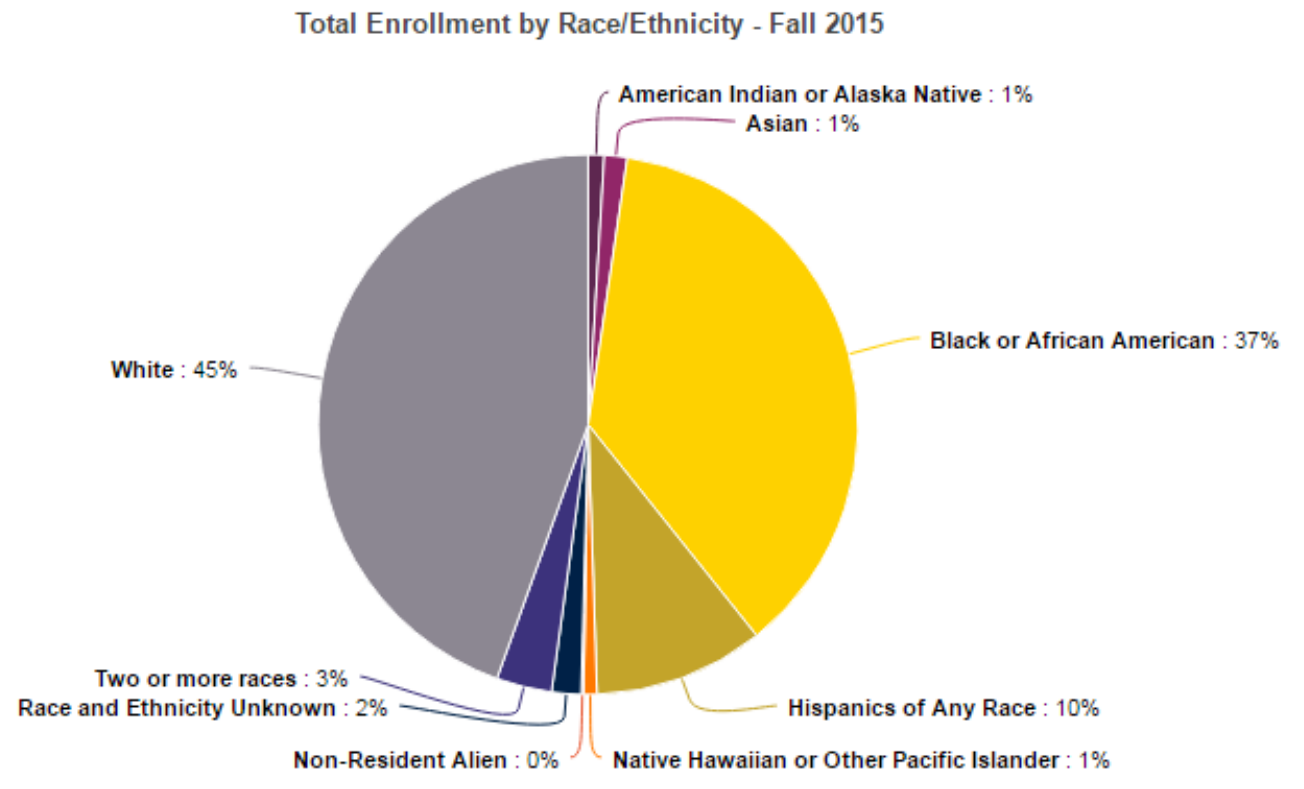

Source: Institutional Research Services, 2015 


\section{Course Model}

All courses utilized within the study were five weeks in length and provided 3 credits, except SCI207 which is a four credit course due to the incorporation of a lab requirement. All courses utilize a standardized design approach where master versions of each course are designed and copied into multiple identical course sections of 30 students or less. This approach allows for a standardized student experience across faculty while allowing for small course enrollment sizes. Each of the courses requires weekly readings, assignments/labs, and mandatory participation in discussion boards. Each discussion board requires the student to post an initial response to a threaded discussion, open to all enrolled students and the instructor, to a specific prompt covering a key concept in the course. Additionally, students are required to respond to multiple peer postings to encourage peer to peer and student to instructor interaction. For courses utilizing lab work, physical lab kits are mailed to the student and hands-on labs are performed within each student's residence.

\section{Experimental Design}

Each week, multiple sections of each course were offered at a maximum enrollment of 30 students per section. During each study week, one-half of the sections for each course were randomly designated as control courses with the second half of sections randomly designated as experimental sections. Control courses received no intervention and ran under normal instructional delivery and faculty requirements. Requirements ensure that instructors interact with at least two new students within the discussion board on at least three separate days of the week. To address the effect of discussion board enrollment size on student success and satisfaction, the discussion board threads for experimental sections were split into smaller cohorts based on the following considerations:

- Courses with 15 or fewer students on the first day of the course remain with one cohort per discussion

- Courses with 16-25 students on the first day of the course are split into two cohorts per discussion

- Courses with 26 or more students on the first day of the course are split into three cohorts per discussion

Each cohort within each course can only see the responses from students within their cohort, creating a discussion board with a much lower enrollment than the overall courses. Instructor requirements for experimental sections remained consistent with the control group with the exception that instructors were required to interact with at least two students within the discussion board on at least three separate days of the week, for each cohort. A number of sections for each course as well as average and total enrollment for each course are available in Table 1. 
Table 1. Course statistics for sections utilized throughout the study.

\begin{tabular}{|c|c|c|c|}
\hline Course & \# Sections & Avg \# Students/Section & Total \# Students \\
\hline \multicolumn{4}{|l|}{ ENG121 } \\
\hline Control & 39 & 26 & 1018 \\
\hline Experimental & 36 & 25 & 911 \\
\hline \multicolumn{4}{|l|}{ ENG122 } \\
\hline Control & 5 & 19 & 93 \\
\hline Experimental & 9 & 20 & 180 \\
\hline \multicolumn{4}{|l|}{ PHI103 } \\
\hline Control & 20 & 28 & 560 \\
\hline Experimental & 17 & 28 & 477 \\
\hline \multicolumn{4}{|l|}{ POL 201 } \\
\hline Control & 15 & 26 & 393 \\
\hline Experimental & 18 & 26 & 468 \\
\hline \multicolumn{4}{|l|}{ SCI207 } \\
\hline Control & 17 & 18 & 299 \\
\hline Experimental & 15 & 18 & 263 \\
\hline \multicolumn{4}{|l|}{ SOC120 } \\
\hline Control & 16 & 31 & 488 \\
\hline Experimental & 15 & 30 & 447 \\
\hline \multicolumn{4}{|l|}{ Total } \\
\hline Control & 112 & 26 & 2886 \\
\hline Experimental & 110 & 25 & 2796 \\
\hline
\end{tabular}

\section{Data Analysis}

Data was compiled from all experimental and control sections and analyzed using SPSS v.23 (SPSS, 2015). To analyze the effectiveness of discussion board cohorts on student success, data was collected for course GPA, withdraw rate (percentage of students withdrawing from the course before the last day), fail rate (percentage of students receiving a grade less than $60 \%$ ), and progression rate (percentage of students attending another course within 2 weeks of completing, withdrawing, or failing the experimental or control course). Interactions between control and experimental groups were analyzed for each course and for all courses combined using one-way ANOVAs.

Additionally, to analyze the effectiveness of discussion board cohorts on student satisfaction, results of student End of Course Surveys (EoCS) were analyzed for both experimental and control groups. Within each course, End of Course Surveys were distributed through email at the end of week 4 to all students enrolled in the course. The survey remained open through the final day of the course at which point it closed to all students. The survey consisted of 16 total questions, nine of which directly evaluated the instructor, five of which directly evaluated the course, and two comprehensive questions (Figure 3). Each question was evaluated by the student utilizing a 5 point scale where $5=$ strongly agree, $4=$ agree, $3=$ neutral/neither agree nor disagree, $2=$ disagree, and $0=$ strongly disagree. For data analysis, answers were coded on a $0-1$ scale where strongly agree $=1$, agree $=0.75$, neutral $/$ neither agree nor disagree $=$ 0.50 , disagree $=0.25$, and strongly disagree $=0$. The response rate for the survey was $41.4 \%$. Interactions between control and experimental groups were analyzed for each course and for all courses combined using one-way ANOVAs. 
Figure 3. End of Course Survey questions distributed to all students enrolled in the study course

\begin{tabular}{|c|c|c|c|c|c|}
\hline \multicolumn{6}{|c|}{ End of Course Survey - Fixed Questions } \\
\hline \multicolumn{6}{|l|}{ Instructor Assessment } \\
\hline $\begin{array}{l}\text { The instructor promotes active } \\
\text { classroom participation of students. }\end{array}$ & $\begin{array}{c}\text { Strongly Agree } \\
\mathbf{4}\end{array}$ & $\begin{array}{c}\text { Agree } \\
\mathbf{3}\end{array}$ & $\begin{array}{l}\text { Neither Agree } \\
\text { nor Disagree } \\
\mathbf{2}\end{array}$ & $\begin{array}{c}\text { Disagree } \\
\mathbf{1}\end{array}$ & $\begin{array}{c}\text { Strongly } \\
\text { Disagree } \\
\mathbf{0}\end{array}$ \\
\hline $\begin{array}{l}\text { The instructor fosters critical thinking } \\
\text { throughout the course. }\end{array}$ & $\begin{array}{c}\text { Strongly Agree } \\
\mathbf{4}\end{array}$ & $\begin{array}{c}\text { Agree } \\
\mathbf{3}\end{array}$ & $\begin{array}{l}\text { Neither Agree } \\
\text { nor Disagree } \\
\mathbf{2}\end{array}$ & $\begin{array}{c}\text { Disagree } \\
\mathbf{1}\end{array}$ & $\begin{array}{c}\text { Strongly } \\
\text { Disagree } \\
\mathbf{0}\end{array}$ \\
\hline $\begin{array}{l}\text { The instructor adds her/his perspective, } \\
\text { such as knowledge and experience, to } \\
\text { the course content. }\end{array}$ & $\begin{array}{c}\text { Strongly Agree } \\
\mathbf{4}\end{array}$ & $\begin{array}{c}\text { Agree } \\
\mathbf{3}\end{array}$ & $\begin{array}{l}\text { Neither Agree } \\
\text { nor Disagree } \\
\mathbf{2}\end{array}$ & $\begin{array}{c}\text { Disagree } \\
\mathbf{1}\end{array}$ & $\begin{array}{c}\text { Strongly } \\
\text { Disagree } \\
\mathbf{0}\end{array}$ \\
\hline $\begin{array}{l}\text { The instructor communicates and } \\
\text { promotes high expectations. }\end{array}$ & $\begin{array}{c}\text { Strongly Agree } \\
\mathbf{4}\end{array}$ & $\begin{array}{c}\text { Agree } \\
\mathbf{3}\end{array}$ & $\begin{array}{l}\text { Neither Agree } \\
\text { nor Disagree } \\
\mathbf{2}\end{array}$ & $\begin{array}{c}\text { Disagree } \\
\mathbf{1}\end{array}$ & $\begin{array}{c}\text { Strongly } \\
\text { Disagree } \\
\mathbf{0}\end{array}$ \\
\hline $\begin{array}{l}\text { The instructor's feedback aligns with } \\
\text { her/his communicated expectations. }\end{array}$ & $\begin{array}{c}\text { Strongly Agree } \\
\mathbf{4}\end{array}$ & $\begin{array}{c}\text { Agree } \\
\mathbf{3}\end{array}$ & $\begin{array}{l}\text { Neither Agree } \\
\text { nor Disagree } \\
\mathbf{2}\end{array}$ & $\begin{array}{c}\text { Disagree } \\
\mathbf{1}\end{array}$ & $\begin{array}{c}\text { Strongly } \\
\text { Disagree } \\
\mathbf{0}\end{array}$ \\
\hline $\begin{array}{l}\text { The instructor provides feedback in a } \\
\text { timely manner. }\end{array}$ & $\begin{array}{c}\text { Strongly Agree } \\
\mathbf{4}\end{array}$ & $\begin{array}{c}\text { Agree } \\
\mathbf{3}\end{array}$ & $\begin{array}{c}\text { Neither Agree } \\
\text { nor Disagree } \\
\mathbf{2}\end{array}$ & $\begin{array}{c}\text { Disagree } \\
\mathbf{1}\end{array}$ & $\begin{array}{c}\text { Strongly } \\
\text { Disagree } \\
\mathbf{0}\end{array}$ \\
\hline $\begin{array}{l}\text { The instructor provides useful feedback } \\
\text { for improving students' quality of work. }\end{array}$ & $\begin{array}{c}\text { Strongly Agree } \\
4\end{array}$ & $\begin{array}{c}\text { Agree } \\
\mathbf{3}\end{array}$ & $\begin{array}{l}\text { Neither Agree } \\
\text { nor Disagree } \\
\mathbf{2}\end{array}$ & $\begin{array}{c}\text { Disagree } \\
\mathbf{1}\end{array}$ & $\begin{array}{c}\text { Strongly } \\
\text { Disagree } \\
\mathbf{0}\end{array}$ \\
\hline $\begin{array}{l}\text { The instructor provides consistent } \\
\text { grading across assignments. }\end{array}$ & $\begin{array}{c}\text { Strongly Agree } \\
\mathbf{4}\end{array}$ & $\begin{array}{c}\text { Agree } \\
\mathbf{3} \\
\end{array}$ & $\begin{array}{l}\text { Neither Agree } \\
\text { nor Disagree } \\
\mathbf{2} \\
\end{array}$ & $\begin{array}{c}\text { Disagree } \\
\mathbf{1} \\
\end{array}$ & $\begin{array}{c}\text { Strongly } \\
\text { Disagree } \\
\mathbf{0} \\
\end{array}$ \\
\hline $\begin{array}{l}\text { I would recommend this instructor to } \\
\text { another student. }\end{array}$ & $\begin{array}{c}\text { Strongly Agree } \\
4\end{array}$ & $\begin{array}{c}\text { Agree } \\
\mathbf{3}\end{array}$ & $\begin{array}{c}\text { Neither Agree } \\
\text { nor Disagree } \\
\mathbf{2} \\
\end{array}$ & $\begin{array}{c}\text { Disagree } \\
\mathbf{1}\end{array}$ & $\begin{array}{c}\text { Strongly } \\
\text { Disagree } \\
\mathbf{0}\end{array}$ \\
\hline $\begin{array}{l}\text { Course assignments require me to think } \\
\text { critically. }\end{array}$ & $\begin{array}{c}\text { Strongly Agree } \\
4\end{array}$ & $\begin{array}{c}\text { Agree } \\
\mathbf{3}\end{array}$ & $\begin{array}{l}\text { Neither Agree } \\
\text { nor Disagree } \\
\mathbf{2}\end{array}$ & $\begin{array}{c}\text { Disagree } \\
\mathbf{1}\end{array}$ & $\begin{array}{c}\text { Strongly } \\
\text { Disagree } \\
\mathbf{0}\end{array}$ \\
\hline $\begin{array}{l}\text { Hard work is required to earn a good } \\
\text { grade in this course. }\end{array}$ & $\begin{array}{c}\text { Strongly Agree } \\
\mathbf{4}\end{array}$ & $\begin{array}{c}\text { Agree } \\
\mathbf{3}\end{array}$ & $\begin{array}{l}\text { Neither Agree } \\
\text { nor Disagree } \\
\mathbf{2}\end{array}$ & $\begin{array}{c}\text { Disagree } \\
\mathbf{1}\end{array}$ & $\begin{array}{c}\text { Strongly } \\
\text { Disagree } \\
\mathbf{0}\end{array}$ \\
\hline $\begin{array}{l}\text { The course content } \\
\text { (assignments/readings/study materials) } \\
\text { is engaging. }\end{array}$ & $\begin{array}{c}\text { Strongly Agree } \\
4\end{array}$ & $\begin{array}{c}\text { Agree } \\
\mathbf{3}\end{array}$ & $\begin{array}{l}\text { Neither Agree } \\
\text { nor Disagree } \\
\mathbf{2}\end{array}$ & $\begin{array}{c}\text { Disagree } \\
\mathbf{1}\end{array}$ & $\begin{array}{c}\text { Strongly } \\
\text { Disagree } \\
\mathbf{0}\end{array}$ \\
\hline $\begin{array}{l}\text { Instructions for completing } \\
\text { assignments are clear. }\end{array}$ & $\begin{array}{c}\text { Strongly Agree } \\
\mathbf{4}\end{array}$ & $\begin{array}{c}\text { Agree } \\
\mathbf{3}\end{array}$ & $\begin{array}{l}\text { Neither Agree } \\
\text { nor Disagree } \\
\mathbf{2}\end{array}$ & $\begin{array}{c}\text { Disagree } \\
\mathbf{1}\end{array}$ & $\begin{array}{c}\text { Strongly } \\
\text { Disagree } \\
\mathbf{0}\end{array}$ \\
\hline $\begin{array}{l}\text { Clear instruction was given on how } \\
\text { assignments would be graded. }\end{array}$ & $\begin{array}{c}\text { Strongly Agree } \\
\mathbf{4}\end{array}$ & $\begin{array}{c}\text { Agree } \\
\mathbf{3}\end{array}$ & $\begin{array}{c}\text { Neither Agree } \\
\text { nor Disagree } \\
\mathbf{2}\end{array}$ & $\begin{array}{c}\text { Disagree } \\
\mathbf{1}\end{array}$ & $\begin{array}{c}\text { Strongly } \\
\text { Disagree } \\
\mathbf{0}\end{array}$ \\
\hline $\begin{array}{l}\text { The quality of my educational } \\
\text { experience has met my expectations. }\end{array}$ & $\begin{array}{c}\text { Strongly Agree } \\
\mathbf{4}\end{array}$ & $\begin{array}{c}\text { Agree } \\
\mathbf{3}\end{array}$ & $\begin{array}{c}\text { Neither Agree } \\
\text { nor Disagree } \\
\mathbf{2}\end{array}$ & $\begin{array}{c}\text { Disagree } \\
\mathbf{1}\end{array}$ & $\begin{array}{c}\text { Strongly } \\
\text { Disagree } \\
\mathbf{0}\end{array}$ \\
\hline $\begin{array}{l}\text { I would recommend this course to } \\
\text { another student. }\end{array}$ & $\begin{array}{c}\text { Strongly Agree } \\
\mathbf{4}\end{array}$ & $\begin{array}{c}\text { Agree } \\
\mathbf{3}\end{array}$ & $\begin{array}{c}\text { Neither Agree } \\
\text { nor Disagree } \\
\mathbf{2} \\
\end{array}$ & $\begin{array}{c}\text { Disagree } \\
\mathbf{1}\end{array}$ & $\begin{array}{c}\text { Strongly } \\
\text { Disagree } \\
\mathbf{0} \\
\end{array}$ \\
\hline
\end{tabular}

\section{RESULTS}

Data showed no significant difference ( $p>0.15$ for all course interactions) in GPA between students participating in one discussion board versus those grouped into smaller cohorts (Figure 4). Similarly, no significant difference was observed in additional student success metrics measured, including average withdraw rate $(p>0.2$ for all course 
interactions; Figure 5), average fail rate ( $p>0.25$ for all course interactions; Figure 6), and average progression rate ( $p>0.05$ for all course interactions, Figure 7) between students participating in one discussion board versus those grouped into smaller cohorts. However, a trend for higher progression for students in discussion cohort sections (96\%) versus those in standard sections $(87 \%)$ was seen for students in ENG122 $(\mathrm{p}=0.06)$.

Similarly, no significant difference could be seen in EoCS responses between students in the control and experimental groups ( $p>0.05$ for all course interactions, Table 2). While not significant at $p<0.05$, EoCS responses did trend lower in experimental sections in comparison with control sections (Table 3).

Figure 4. Average GPA for pilot vs. control sections. $\mathrm{n}=112$ sections for control and 110 sections for experimental courses, $\mathrm{n}=$ 2886 total students for the control courses and 2796 total students for the experimental courses, $p>0.05$ for all control vs experimental course interactions (One-way ANOVAs)

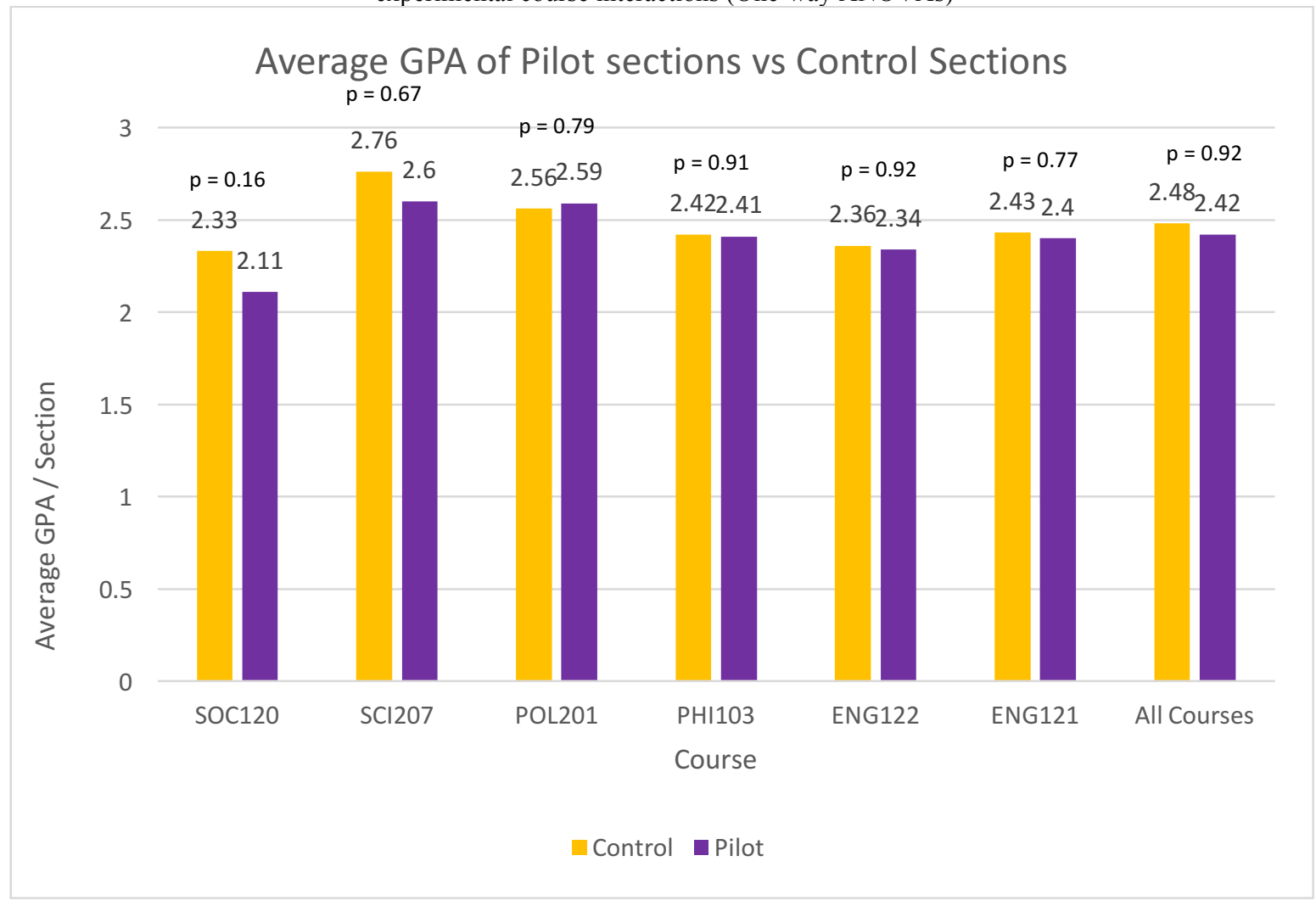


Figure 5. Average withdraw rate (\%) for pilot vs. control sections. $n=112$ sections for control and 110 sections for experimental courses, $n=2886$ total students for the control courses and 2796 total students for the experimental courses, $p>0.05$ for all control vs experimental course interactions (One-way ANOVAs)

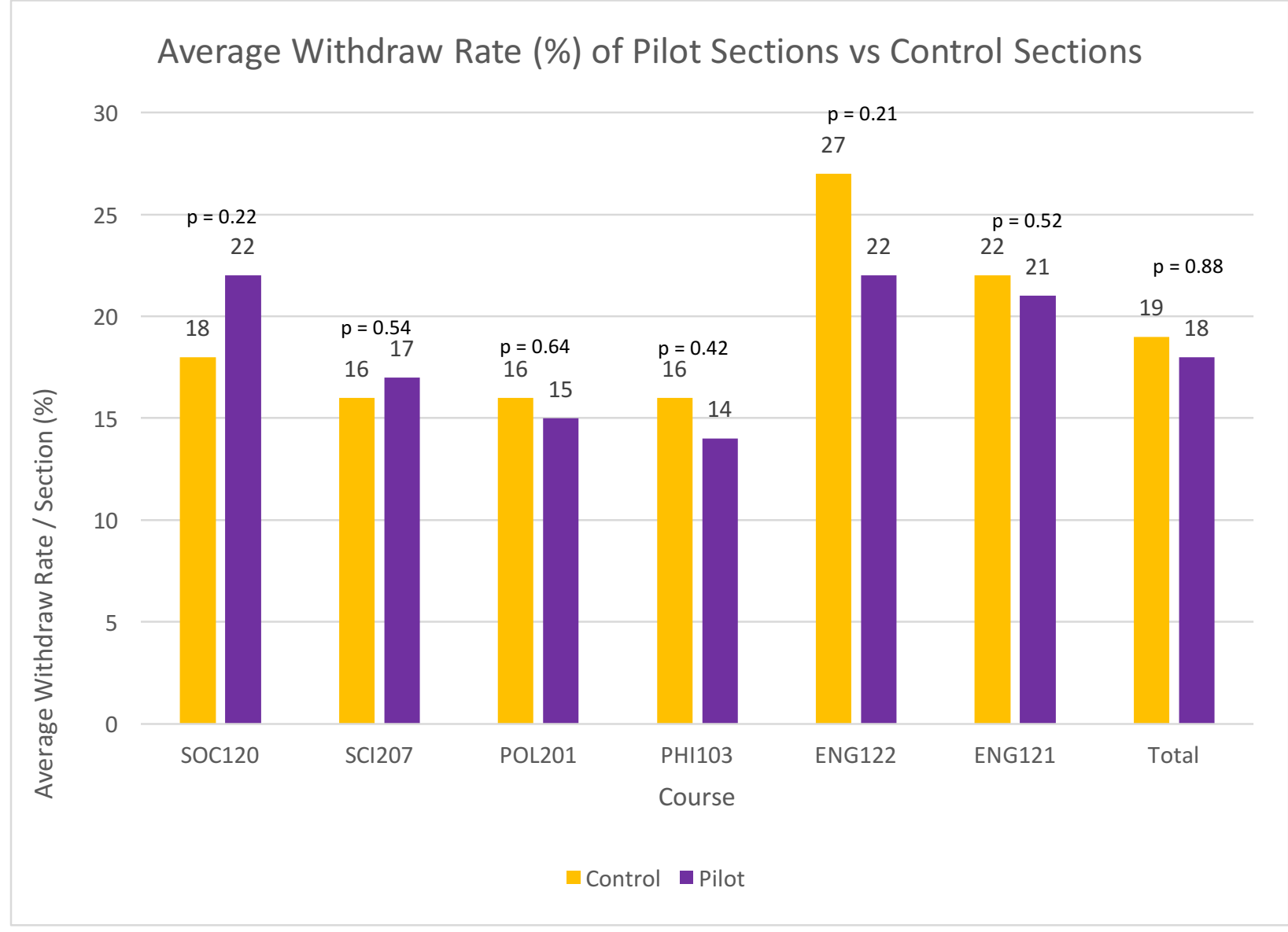


Figure 6. Average fail rate (\%) for pilot vs. control sections. $\mathrm{n}=112$ sections for control and 110 sections for experimental courses, $\mathrm{n}=2886$ total students for the control courses and 2796 total students for the experimental courses, $\mathrm{p}>0.05$ for all control vs experimental course interactions (One-way ANOVAs)

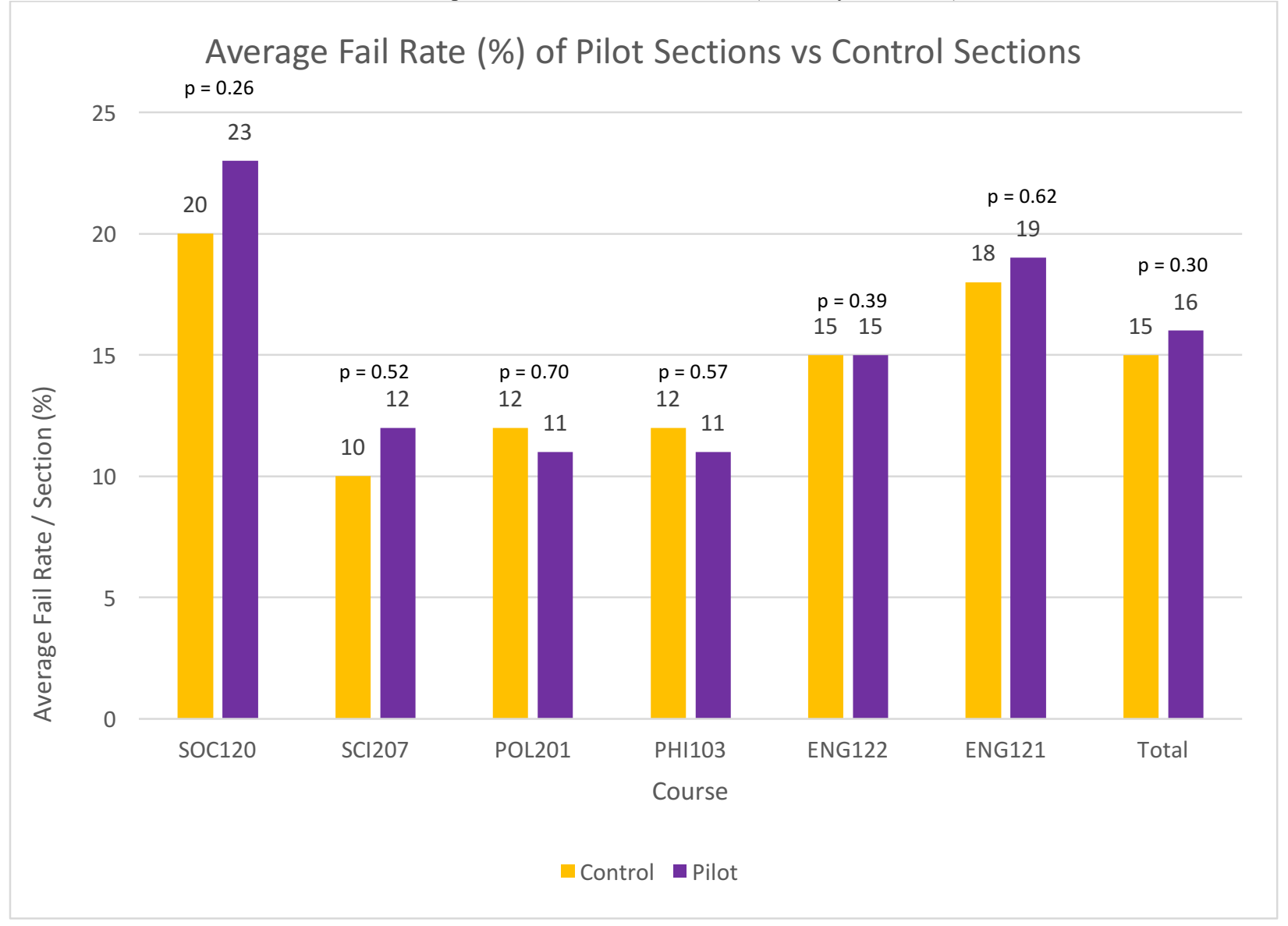


Figure 7. Average progression rate (\%) for pilot vs. control sections. $n=112$ sections for control and 110 sections for experimental courses, $\mathrm{n}=2886$ total students for the control courses and 2796 total students for the experimental courses, $\mathrm{p}>0.05$ for all control vs experimental course interactions (One-way ANOVAs)

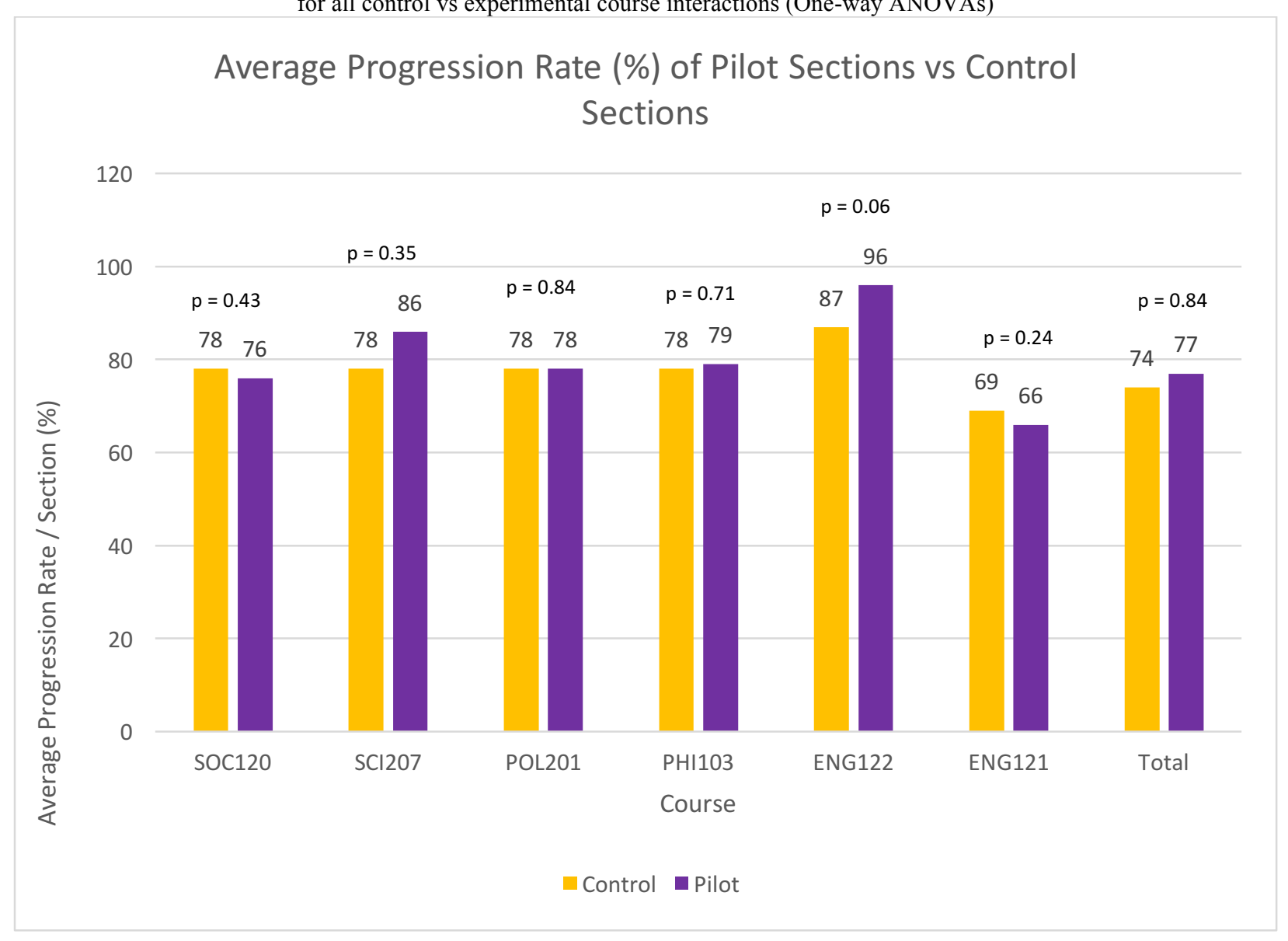


Table 2. Average End of Course Survey responses for individual study courses and questions. $\mathrm{n}=5-39$ sections for control courses and 9-36 for experimental courses, $\mathrm{n}=93-1018$ students for control courses and 180-911 students for experimental courses, $\mathrm{p}>0.05$ for all control vs experimental interactions (One-way ANOVAs).

\begin{tabular}{|c|c|c|c|c|c|c|c|c|c|c|c|c|}
\hline \multirow{3}{*}{ EoCS Question } & \multicolumn{2}{|c|}{ SOC102 } & \multicolumn{2}{|c|}{ SCI207 } & \multicolumn{2}{|c|}{ POL201 } & \multicolumn{2}{|c|}{ PHI103 } & \multicolumn{2}{|c|}{ ENG122 } & \multicolumn{2}{|c|}{ ENG121 } \\
\hline & Con & $\operatorname{Exp}$ & Con & $\operatorname{Exp}$ & Con & $\operatorname{Exp}$ & Con & $\operatorname{Exp}$ & Con & $\operatorname{Exp}$ & Con & $\operatorname{Exp}$ \\
\hline & \multicolumn{12}{|c|}{$(0-1)$} \\
\hline $\begin{array}{l}\text { Instructor Assessment } \\
\text { (Instructor...) }\end{array}$ & & & & & & & & & & & & \\
\hline promotes active participation & .86 & .85 & .89 & .89 & .91 & .89 & .95 & .91 & .90 & .88 & .91 & .88 \\
\hline fosters critical thinking & .86 & .85 & .89 & .90 & .91 & .90 & .95 & .91 & .90 & .89 & .91 & .89 \\
\hline adds experience to course & .82 & .83 & .88 & .89 & .89 & .89 & .95 & .86 & .87 & .86 & .89 & .87 \\
\hline promotes high expectations & .84 & .85 & .88 & .90 & .91 & .90 & .94 & .89 & .89 & .88 & .90 & .87 \\
\hline feedback aligns w/ expectations & .82 & .81 & .88 & .87 & .88 & .86 & .94 & .85 & .87 & .86 & .90 & .86 \\
\hline Provides timely feedback & .79 & .80 & .84 & .85 & .86 & .84 & .93 & .83 & .82 & .84 & .85 & .82 \\
\hline Provides useful feedback & .80 & .80 & .87 & .86 & .85 & .83 & .93 & .84 & .85 & .87 & .89 & .86 \\
\hline Provides consistent grading & .82 & .82 & .88 & .87 & .87 & .87 & .93 & .85 & .88 & .86 & .88 & .86 \\
\hline I would recommend this instructor & .78 & .76 & .87 & .85 & .83 & .84 & .92 & .82 & .87 & .82 & .88 & .84 \\
\hline \multicolumn{13}{|l|}{$\begin{array}{l}\begin{array}{l}\text { Course } \\
\text { course...) }\end{array} \\
\text { Assessment }\end{array}$} \\
\hline assignments require critical thinking & .89 & .89 & .90 & .90 & .91 & .92 & .96 & .89 & .87 & .91 & .92 & .90 \\
\hline required hard work for a high grade & .91 & .91 & .91 & .93 & .92 & .92 & .95 & .90 & .90 & .92 & .93 & .91 \\
\hline content is engaging & .81 & .80 & .86 & .87 & .84 & .83 & .86 & .82 & .80 & .80 & .85 & .81 \\
\hline Instructions are clear & .84 & .83 & .90 & .89 & .88 & .88 & .92 & .87 & .86 & .85 & .89 & .88 \\
\hline Grading criteria were clear & .78 & .77 & .86 & .84 & .86 & .86 & .88 & .83 & .82 & .80 & .84 & .81 \\
\hline \multicolumn{13}{|l|}{ Comprehensive Items } \\
\hline Qual of experience met expectations & .78 & .78 & .87 & .84 & .83 & .85 & .89 & .82 & .86 & .83 & .87 & .83 \\
\hline I would recommend this course & .76 & .75 & .85 & .82 & .81 & .84 & .85 & .81 & .82 & .79 & .87 & .82 \\
\hline
\end{tabular}

Table 3. Average End of Course Survey responses for all study courses and questions. $n=112$ sections for control courses and 110 for experimental courses, $\mathrm{n}=2886$ students for control courses and 2796 students for experimental courses, $\mathrm{p}>0.05$ for all control vs experimental interactions (One-way ANOVAs).

\begin{tabular}{l|c|c}
\hline \multicolumn{1}{c}{ EoCS Question } & \multicolumn{2}{c}{ All Courses } \\
\hline Instructor Assessment (Instructor...) & Control (\%) & Experimental (\%) \\
\hline promotes active participation & & \\
\hline fosters critical thinking & 90 & 88 \\
\hline adds experience to course & 90 & 89 \\
\hline promotes high expectations & 87 & 87 \\
\hline feedback aligns w/ expectations & 89 & 85 \\
\hline Provides timely feedback & 88 & 83 \\
\hline Provides useful feedback & 84 & 85 \\
\hline Provides consistent grading & 86 & 86 \\
\hline I would recommend this instructor & 87 & 83 \\
\hline Course Assessment (The course...) & 85 & \\
\hline assignments require critical thinking & & 90 \\
\hline required hard work for a high grade & 91 & 92 \\
\hline content is engaging & 92 & 82 \\
\hline Instructions are clear & 84 & 87 \\
\hline Grading criteria were clear & 88 & 82 \\
\hline Comprehensive Items & 84 & 83 \\
\hline Qual of experience met expectations & & 81 \\
\hline I would recommend this course & 85 & 83 \\
\hline
\end{tabular}




\section{DISCUSSION/CONCLUSIONS}

The study explored the relationship between cohort size in discussions and student success metrics as well as student perceptions of the courses. Contrary to the hypotheses, analyses revealed that there were no significant differences in student success metrics between courses of varying discussion cohort sizes. Also, although not statistically significant, indirect measures (End of Course Survey) indicated a trend for enhanced student satisfaction in courses with larger discussions and less direct interaction with faculty members. These outcomes seem to contradict literature that indicates that student learning and perceived experience of the courses are positively influenced by the size of the discussions (Barkley, Cross, and Major, 2005 \& Du, Durrington, and Matthews, 2007)

Smaller discussion forums with more faculty participation did not lead to any statistically significant differences in GPA, withdrawal rate, fail rate, or progression rate across all tested courses. On an individual course level, there was one statistically significant positive difference in the progression rate of students in ENG 122 (Intermediate Composition). Because all students complete the same written assignments in specific Ashford courses, and these assignments are linked directly to learning outcomes, the lack of improvement in GPA indicates that greater faculty engagement in smaller discussion cohorts had no significantly greater positive impact on student learning.

In relation to the EoCS activity outcomes, it is of special interest to note that students in smaller cohorts tended to track lower in their responses to the following questions:

- Instructor promotes active participation

- Instructor fosters critical thinking

- Instructor provides useful feedback

- Quality of experience met my expectations

Even in courses with three times as much faculty participation and interaction with students in the discussion forums, the EOCS outcomes tracked negatively in the experimental sections of the courses, although these differences were not statistically significant. In this case, increased faculty engagement in the discussion forums does not align with perceived engagement or satisfaction in the students themselves. Also, students also did not perceive any improvement in the facilitation of critical thinking, an area that many online teachers believe is best developed in the discussions.

Greater levels of interaction with faculty in smaller discussion cohorts did not facilitate greater levels of learning, successful completion, or retention. At the very least it was believed that student perceptions of the faculty and the courses would be more positive about faculty engagement questions and facilitation of thinking. However, the evidence indicates that students prefer courses with larger groups of their peers and less direct interaction with their professors. Also, students' success metrics in larger courses with less direct interaction with faculty were in conformity with courses with smaller discussions and more engagement.

It is important to note that this study focused on the lower level general education courses. While students may take general education courses throughout their careers most do so early in their tenure at Ashford. While one might believe that greater levels of direct faculty interaction in discussions with smaller groups of students would be welcome and engaging, from a different perspective, this might be threatening and intimidating. Students in general education courses that are not directly aligned to their major programs might prefer larger groups of peers and more interactions with their peers. The larger group setting allows for more anonymity for those students who do not feel comfortable in the discussion forums. In certain contexts, and with certain groups of students, teachers can be perceived as threatening and direct interactions can lead to fear and a sense of inadequacy despite the teacher's best attempts to establish a welcoming learning environment. Students in larger discussions might also enjoy interactions with their peers and find those interactions more supportive and engaging. This could lead to secondary perceptions of the teacher and the course as being more positive and facilitating greater levels of interaction between students, thus ending in a more positive perception of the instructor's interactions.

The demographics of the student body at Ashford are important in understanding the outcomes of this study as well. Some literature indicates that certain models of discussions lead to more positive outcomes for students. However, 
these groups often represent different demographic groups. The ways in which adults interact in these forums and their beliefs about the necessity of discussions in the first place might limit the importance that they place on these interactions and the learning that takes place in them (Panacci, 2015).

Online discussion boards are an integral part of any online program or curriculum. Knowing how to place students in groups to facilitate enhanced learning and student success is extremely important for teachers and institutions. Smaller discussion cohorts require more people and more time. In addition, this study indicates that in certain contexts this actually has no impact on important aspects of student learning. There is a tendency to think that smaller class sizes or groups facilitate better learning experiences for students. While this might be the case in specific instances, more work needs to be done in online learning to determine where larger course sizes might be better. Since the number of interactions in the discussions had no overall positive impact on student learning or their perceptions of the quality of the courses and instructors, future research should focus on qualitative analysis of individual faculty behaviors in the discussions as well as the content of the discussion forum prompts themselves to determine efficacy in relation to learning, retention, and student satisfaction.

\section{AUTHOR BIOGRAPHIES}

Dr. Adam Selhorst is the Executive Dean of the College of Liberal Arts at Ashford University. Dr. Selhorst holds a $\mathrm{PhD}$ and MS in Environmental Science from the Ohio State University, an MA in Public Policy and Management from the Ohio State University, an MBA in Entrepreneurship from Ashford University, and a BA in Biology from Kenyon College. His previous academic experience includes positions as a Fellow at the National Science Foundation, Lecturer at the Ohio State University, Lecturer at Leeward Community College, Researcher at the University of Hawaii, and Student Research Scientist at Kenyon College.

Dr. Eric Klein is an Associate Dean in the College of Liberal Arts at Ashford University. Dr. Klein earned his PhD in Clinical Psychology from Fairleigh Dickinson University and completed his pre-doctoral internship at the University of South Carolina. Dr. Klein is actively involved in several professional and community organizations, as he currently serves on multiple committees for the American Psychological Association, and serves on the Alcohol and Drug Services Advisory Board for San Diego County.

Dr. Justin Harrison is the Dean of the Division of General Education at Ashford University. He received his BA in Biology from Taylor University, an MA in Philosophy at the University of Toledo, and his PhD at Loyola University, where he focused on 20th century French and German philosophy, specifically phenomenology and radical empiricism.

\section{REFERENCES}

Arbaugh, J. B., \& Benbunan-Fich, R. (2006). An investigation of epistemological and social dimensions of teaching in online learning environments. Academy of Management Learning \& Education, 5, 435-447.

Barkley, E., Cross, K., \& Major, C. (2005). Collaborative learning techniques: A Handbook for college faculty. San Francisco: Jossey-Bass.

Berry, G. (2008). Asynchronous discussions: Best practices. In 24th Annual Conference on Distance Teaching \& Learning. Madison: University of Wisconsin System. Retrieved from http://www.uwex.edu/disted/conference/Resource_library/proceedings/08_12701.pdf

Buckingham, S. (2003). Perspectives on the experience of the learning community through online discussions. Journal of Distance Education, 18, 74-91.

Burruss, N. M., Billings, D. M., Brownrigg, V., Skiba, D. J., \& Connors, H. R. (2009). Class sizeas related to the use of technology, educational practices, and outcomes in web-based nursing courses. Journal of Professional Nursing, 25 , $33-41$.

Dykman, C. A., \& Davis, C. K. (2008). Online education forum, part three. A quality online educational experience. Journal of Information Systems Education, 19, 281-289.

Du, J., Durrington, V. A., \& Matthews, J. G. (2007). Online collaborative discussion: Myth or valuable learning tool. Journal of Online Learning and Teaching, 3, $94-104$.

Fernandez, M. L. (2007). Communication and instruction in an online graduate education course. Teaching Education, 18, 137150 . 
Fisher, M., Thompson, G. S., \& Silverberg, D. A. (2005). Effective group dynamics in e-learning: Case study. Journal of Educational Technology Systems, 33, 205-222.

Huang, H. \& Liaw, S. (2004). Guiding distance educators in building web-based instructions. International Journal of Instructional Media, 31, 125-137.

Keeton, M. T. (2004). Best online instructional practices: Report of Phase 1 of an ongoing study. Journal of Asynchronous Learning Networks, 8, 75-100.

Levin, B., He, Y., \& Robbins, H. (2006). Comparative analysis of preservice teachers' reflective thinking in synchronous versus asynchronous online case discussions. Journal of Technology and Teaching Education, 14, 439-460.

Marks, R. B., Sibley, S. D., \& Arbaugh, J. B. (2005). A structural equation model of predictors for effective online learning. Journal of Management Education, 29, 531-63.

Panacci, A. G. (2015). Adult students in higher education: Classroom experiences and needs. College Quarterly, 18(3), 1-17. Retrieved from http://files.eric.ed.gov/fulltext/EJ1087330.pdf

Schellens, T. \& Valcke, M. (2006). Fostering knowledge construction in university students through asynchronous discussion groups. Computers and Education, 46, 349-370.

Schutt, M., Allen, B. S., \& Laumakis, M. A. (2009). The effects of instructor immediacy behaviors in online learning environments. Quarterly Review of Distance Education, 10, 135-148. 